\begin{tabular}{c|c|c|} 
SELECCIONES MATEMÁTICAS \\
Universidad Nacional de Trujillo \\
ISSN: 2411-1783 (Online) \\
2021; Vol. 8(1): 66-74.
\end{tabular}

\title{
A new decomposition of open and closed sets in topological spaces
}

\author{
Carlos Granados ${ }^{\circledR}$
}

Received, Jun. 05, 2020

Accepted, Feb. 16, 2021

How to cite this article:

Granados C. A new decomposition of open and closed sets in topological spaces. Selecciones Matemáticas. 2021;8(1):6674. http://dx.doi.org/10.17268/sel.mat.2021.01.06

\begin{abstract}
In this paper, we show some properties on ${ }^{\star} b$-open sets. Besides, we introduce the notions of generalized ${ }^{\star} b$ closed sets, locally ${ }^{\star} b$-closed sets, regular generalized ${ }^{\star} b$-closed sets and weakly ${ }^{\star} b$-closed sets. Moreover, we study some of their properties.

Keywords . ${ }^{\star} b$-open sets, Generalized ${ }^{\star} b$-closed sets, Locally ${ }^{\star} b$-closed sets, Regular generalized ${ }^{\star} b$-closed sets, Weakly ${ }^{\star} b$-closed sets.
\end{abstract}

1. Introduction and preliminaries. A topological space is well-known as follows $(X, \tau)$, where $\tau$ is the topology on $X$. Mathematicials have developed an interested of studying open and closed sets in topological spaces; in 2012 Indira and Rekha [9] introduced the concept of ${ }^{\star} b$-open set as a generalization for introducing the notion of ${ }^{\star \star} b$-open set, but they did not show many results, for that reason, in this paper, we took that idea and we extend this notion and show some new properties on ${ }^{\star} b$-open sets. Nevertheless, the results which were introduced by them and are necessary for the developing of this paper can be seen in Definition 2.1 and Theorem 2.1, for more details see [9]. Additionally, we introduce and study some characterization on ${ }^{\star} b$-closed sets which are namely generalized of ${ }^{\star} b$-closed sets, locally ${ }^{\star} b$-closed sets, regular generalized ${ }^{\star} b$-closed, weakly ${ }^{\star} b$-closed sets. Moreover, we show some of their properties and some relations among them.

Otherwise, studying open an closed sets in general topology is increising in several fields on topology; Hariwan [7], introduced the notion of $B c$-open set in a topological spaces for extending the concept of $b$ open set in which every $b$-open set is $B c$-open. Taking into account mentioned above, in this paper section two, we proved that every ${ }^{\star} b$-open set is $b$-open, indeed every ${ }^{\star} b$-open set is $B c$-open.

On the other hand, the notions of open and closed sets which are introducing in this paper, it will help to introduce others notions such that ${ }^{\star} b$-open sets in bitopological or tritopological spaces, generalized by using the notion of $\omega$-closed sets [8], generalized by using the notion of $\Lambda$-sets [14], ${ }^{\star} b$-open sets in ideal topological spaces [10], nano topological spaces [4], operator interior $\psi$ [5], convergence spaces [6] and so on.

This paper is divided in six section, in the first one, we show preliminaries in which we show some well-known notions which are useful for the developing this paper; in the second on, we study and extend the notions of ${ }^{\star} b$-open and ${ }^{\star} b$-closed set; in the third until the sixth, we show some characterizations on ${ }^{\star} b$-closed sets.

Throughout this paper, $(X, \tau)$ is a topological spaces on which no separation axioms are assumed unless otherwise mentioned. Besides, we sometimes write $X$ instead $(X, \tau)$. Now, we show some notions which are useful for the developing of this paper.

Definition 1.1. Let $A$ be a subset of a topological space $X$, then $A$ is said to be semi-open [11] if

*Universidad del Atlántico, Barranquilla-Colombia (carlosgranadosortiz@outlook. es). 
$A \subseteq C l(\operatorname{Int}(A))$.

Definition 1.2. Let $A$ be a subset of a topological space $X$, then $A$ is said to be pre-open [13] if $A \subseteq \operatorname{Int}(C l(A))$.

Definition 1.3. Let $A$ be a subset of a topological space $A$, Then $A$ is said to be b-open [2] if $A \subseteq$ $C l(\operatorname{Int}(A)) \cup \operatorname{Int}(C l(A))$.

Theorem 1.1. [2] Let $A$ be a subset of a topological space $X$, then the following statements hold:

1. If $A$ is a semi-open set, then $A$ is a b-open set.

2. If $A$ is pre-open set, then $A$ is a b-open set.

Definition 1.4. The union of all semi-open and pre open sets of $X$ contain in $A$ is called semi-interior and pre-interior of $A$ and it is denoted by [11] $\operatorname{sint}(A)$ and [13] pInt $(A)$, respectively.

Definition 1.5. A subset $A$ of a topological space $X$ is said to be regular-open [15] if $A=\operatorname{Int}(C l(A))$.

Definition 1.6. A subset $A$ of a space $X$ is called locally b-closed [1] if $A=U \cap V$, where $U \in \tau$ and $V$ is a b-closed set.

Definition 1.7. A generalized pre regular closed set simply gpr-closed [3] if $p C l(A) \subseteq U$ whenever $A \subseteq U$ and $U$ is a regular open in $X$.

Definition 1.8. A generalized pre regular closed set simply rgb-closed [12] if $b C l(A) \subseteq U$ whenever $A \subseteq U$ and $U$ is a regular open in $X$.

Definition 1.9. A subset $A$ of $(X, \tau)$ is called nowhere dense [16] if $\operatorname{Int}(C l(A))=\emptyset$.

2. ${ }^{\star} b$-open sets. In this section, we show some new properties on ${ }^{\star} b$-open sets.

Definition 2.1. Let $(X, \tau)$ be a topological space and $A \subseteq X$. Then, $A$ is said to be ${ }^{\star} b$-open [9] if $A \subseteq C l(\operatorname{Int}(A)) \cap \operatorname{Int}(C l(A))$. The complement of $a^{\star} b$-open set is called ${ }^{\star} b$-closed set.

Remark 2.1. The collection of all ${ }^{\star} b$-open sets and ${ }^{\star} b$-closed sets are denoted by $B S O(X, \tau)$ and $B S O(X, \tau)$, respectively.

Lemma 2.1. Every open set of $X$ is $a^{\star} b$-open set of $X$.

Proof: Let $A$ be a open set of $X$. Then, $A \subseteq C l(\operatorname{Int}(A)) \cap \operatorname{Int}(C l(A))$, since $A$ is an open set, then $\operatorname{Int}(A)=A$ and $C l(A)=X$ or $C l(A)=B$ where $B$ is an closed set of $X$. Thus, $A \subseteq C l(A) \cap \operatorname{Int}(B)$. Now, $C l(A)=X$ or $C l(B)$ where $B$ is an closed set of $X$ and $A \subseteq \operatorname{Int}(B)$, therefore $\operatorname{Int}(B)=A$ or $\operatorname{Int}(B)=C$ where $A \subseteq C \subseteq \operatorname{Int}(B)$. In consequence, $A \subseteq B \cap C$, but $A \subseteq B$ and $A \subseteq C$, this implies that $A \subseteq B \cap C$. Hence $A$ is a ${ }^{\star} b$-open set of $X$. The following example shows that the converse of the above Lemma, it is not always true.

Example 2.1. Let $X=\{a, b, c, d\}$ and $\tau=\{\emptyset, X,\{a\},\{b\},\{a, b\},\{a, b, d\}\}$. Then, $\{a, b, c\}$ is $a$ ${ }^{\star}$ b-open set, but it is not an open set.

Theorem 2.1. Let $(X, \tau)$ be a topological space. If $\left\{A_{\delta}: \delta \in \Delta\right\}$ is a family of ${ }^{\star} b$-open set, [9] then $\bigcup_{\delta \in \Delta} A_{\delta}$ is ${ }^{\star}$ b-open.

Corollary 2.1. Let $(X, \tau)$ be a topological space. If $\left\{A_{\delta}: \delta \in \Delta\right\}$ is a family of ${ }^{\star} b$-closed set, then $\bigcap A_{\delta}$ is ${ }^{\star}$-closed. Proof: It follows from the Theorem 2.1 .

Definition 2.2. Let $(X, \tau)$ be a topological space and $A \subset X$. An element $x \in A$ is said to be ${ }^{\star} b$-interior point of $A$ if there exits $a^{\star} b$-open set $U$ such that $x \in U \subseteq A$. The set of all ${ }^{\star} b$-interior points of $A$ is said to be ${ }^{\star} b$-interior of $A$ an it is denoted by ${ }^{\star} b$ - Int $(A)$.

Lemma 2.2. If $A$ is a subset of $(X, \tau)$, then ${ }^{\star} b-\operatorname{Int}(A)=\operatorname{sInt}(A) \cap p \operatorname{Int}(A)$.

Proof: The proof is followed by the Definitions 1.4 and 2.2.

Theorem 2.2. Let $(X, \tau)$ be a topological space and $A \subset X$. Then, ${ }^{\star} b-\operatorname{Int}(A)=\bigcup\left\{U \mid U\right.$ is ${ }^{\star} b$-open and $U \subseteq A\}$.

Proof: Let $x \in^{\star} b$ - $\operatorname{Int}(A)$. Then, there exits ${ }^{\star} b$-open set $U$ such that $x \in U \subset A$. Hence, $x \in \bigcup\{U \mid U$ is ${ }^{\star} b$-open and $\left.U \subseteq A\right\}$. Then, $y \subset U_{0}$ for some ${ }^{\star} b$-open set $U_{0} \subset A$. Therefore, $y \in^{\star} b$ - $\operatorname{Int}(A)$. In consequence, $\bigcup\left\{U \mid U\right.$ is ${ }^{\star} b$-open and $\left.U \subset A\right\} \subseteq \subseteq^{\star} b$ - $\operatorname{Int}(A)$.

Theorem 2.3. Let $(X, \tau)$ be a topological space and $A \subset X$. Then, $A$ is ${ }^{\star} b$-open if and only if $A={ }^{\star} b-\operatorname{Int}(A)$.

Proof: Let $A$ be a ${ }^{\star} b$-open set. Then, $A \subseteq A$ and this implies that $A \in\left\{U \mid U\right.$ is ${ }^{\star} b$-open and $\left.U \subset A\right\}$. Since union of this collection is in $A$. Therefore, $A={ }^{\star} b-\operatorname{Int}(A)$. Conversely, suppose that $A={ }^{\star} b-\operatorname{Int}(A)$. Hence, $A$ is ${ }^{\star} b$-open.

Definition 2.3. Let $A \subset X$. Then $x \in X$ is ${ }^{\star} b$-adherent to $A$ if $U \cap A \neq \emptyset$ for every ${ }^{\star} b$-open set $U$ containing $x$. The set of all ${ }^{\star} b$-adherent points of $A$ is said to be ${ }^{\star} b$-closure of $A$ and it is denoted by 
${ }^{\star} b-C l(A)$.

Theorem 2.4. Let $(X, \tau)$ be a topological space and $A, B \subset X$. Then, the following statements hold:

1. $A \subseteq{ }^{\star} b-C l(A)$.

2. ${ }^{\star} b$ - $C l(A)$ is the smallest ${ }^{\star} b$-closed set containing $A$, that is ${ }^{\star} b-C l(A)=\bigcap\left\{W \mid W\right.$ is ${ }^{\star} b$-closed and $A \subseteq W\}$.

3. $A$ is ${ }^{\star} b$-closed if and only if $A={ }^{\star} b-C l(A)$.

4. If $A \subseteq B$, then ${ }^{\star} b-C l(A) \subseteq{ }^{\star} b-C l(B)$.

5. ${ }^{\star} b-C l(A) \cup{ }^{\star} b-C l(B) \subseteq \subseteq^{\star} b-C l(A \cup B)$.

6. ${ }^{\star} b-C l(A \cap B) \subseteq{ }^{\star} b-C l(A) \cap{ }^{\star} b-C l(B)$.

Proof:

1. Let $x \in A$ and supposes that $x \notin^{\star} b-C l(A)$. Then, there exits ${ }^{\star} b$-open set $V$ containing $x$ such that $V \cap A=\emptyset$ and this is a contradiction. Therefore, $x \in^{\star} b-C l(A)$.

2. Let $x \in^{\star} b-C l(A)$. Then, $V \cap A \neq \emptyset$ for every ${ }^{\star} b$-open set $V$ containing $x$. Now, suppose the contrary, that

$$
x \notin \bigcap\left\{W \mid W \text { is }{ }^{\star} b \text {-closed and } A \subseteq W\right\} .
$$

Then, $x \notin W$ for some ${ }^{\star} b$-closed set $W$, so $x \in X-W$ for some ${ }^{\star} b$-open set $X-W$. So, $(X-W) \cap A=\emptyset$ for some ${ }^{\star} b$-open set $X-W$ containing $x$ and this is a contradiction. Therefore, $x \notin \bigcap\left\{W \mid W\right.$ is ${ }^{\star} b$-closed and $\left.A \subseteq W\right\}$. Conversely, let $y \in x \notin \bigcap\left\{W \mid W\right.$ is ${ }^{\star} b$-closed and $A \subseteq W\}$. Then, $y \in W$ for all ${ }^{\star} b$-closed set $W$ containing $A$. Now, suppose that $y \notin^{\star} b-C l(A)$. Then, there exits ${ }^{\star} b$-open set $V$ containing $y$ such that $V \cap A=\emptyset$. Therefore, $X-V$ is ${ }^{\star} b$-closed set containing $A$ and $y \notin X-V$ and this is a contradiction. Therefore, $y \in^{\star} b-C l(A)$.

The proof of (3) and (4) are followed directly from the Definition 2.3. (5) and (6) are followed by applying part (4) of this Theorem.

Theorem 2.5. Let $(X, \tau)$ be a topological space and $A \subseteq X$. If $A$ is $a^{\star} b$-open set, then $A$ is $a$ semi-open set.

Proof: Let $A$ be a ${ }^{\star} b$-open set, then

$A \subseteq C l(\operatorname{Int}(A)) \cap \operatorname{Int}(C l(A))$,

$A \subset C l(\operatorname{Int}(A))$.

Therefore, $A$ is a semi-open set.

The following example shows that the converse of the above Theorem, it is not always true.

Example 2.2. Let $X=\{a, b, c\}$ and $\tau=\{\emptyset, X,\{a\},\{b\},\{a, b\}\}$. Then, $\{b, c\}$ is a semi-open set, but it is not $a^{\star} b$-open set.

Theorem 2.6. Let $(X, \tau)$ be a topological space and $A \subseteq X$. If $A$ is $a^{\star} b$-open set, then $A$ is a pre-open set.

Proof: Let $A$ be a ${ }^{\star} b$-open set, then

$A \subseteq C l(\operatorname{Int}(A)) \cap \operatorname{Int}(C l(A))$,

$A \subset \operatorname{Int}(C l(A))$.

Therefore, $A$ is a pre-open set.

Remark 2.2. The converse of the above Theorem is not always true when $C l(\operatorname{Int}(A))=\emptyset$ and $\operatorname{Int}(C l(A))=B$, where $A \subseteq B$.

Proposition 2.1. Every ${ }^{\star} b$-open set is a b-open set.

Proof: The proof is followed by the Theorem 1.1, 2.5 and 2.6.

Theorem 2.7. The intersection of a b-open set and $a^{\star} b$-open set is a b-open set.

Proof: Let $A$ be a ${ }^{\star} b$-open set and $B$ be a $b$-open set. Then

$$
\begin{aligned}
A \cap B & \subset[C l(\operatorname{Int}(A)) \cap \operatorname{Int}(C l(A))] \cap[C l(\operatorname{Int}(B)) \cup \operatorname{Int}(C l(B))] \\
& =[C l(\operatorname{Int}(A)) \cap \operatorname{Int}(C l(A)) \cap C l(\operatorname{Int}(B))] \cup[\operatorname{Int}(C l(A) \cap C l(\operatorname{Int}(A)) \cap \operatorname{Int}(C l(B))] \\
& \subset[\operatorname{Int}(C l(A)) \cap C l(\operatorname{Int}(A \cap B))] \cup[C l(\operatorname{Int}(A)) \cap \operatorname{Int}(C l(A \cap B))] .
\end{aligned}
$$

By The Proposition 2.1, $A \cap B$ is a $b$-open set and let $A \cap B=C$. Then

$$
[\operatorname{Int}(C l(A)) \cap C l(\operatorname{Int}(C))] \cup[C l(\operatorname{Int}(A)) \cap \operatorname{Int}(C l(C))] .
$$

Now, $\operatorname{Int}(C l(A)) \cap C l(\operatorname{Int}(C))=D$, where $D \neq \emptyset$ and $C l(\operatorname{Int}(A)) \cap \operatorname{Int}(C l(C))=E$, where $E \neq \emptyset$, and so.

$A \cap B \subseteq D \cup E$, where $D \cup E$ is a $b$-open set.

Definition 2.4. Let $(X, \tau)$ be a topological space and $A \subset X$. Then, $A$ is said to be ${ }^{\star} b$-set if $A=$ $C l(\operatorname{Int}(A)) \cap \operatorname{Int}(C l(A))$.

Remark 2.3. The collection of all ${ }^{\star} b$-set is denoted by $B E S(X, \tau)$. The following example shows that the union of two ${ }^{\star} b$-sets not need be a ${ }^{\star} b$-set. 
Example 2.3. Let $X=\{a, b, c\}$ and $\tau=\{\emptyset, X,\{a\},\{b\},\{a, b\}\}$. The collection of $B E S(X, \tau)=$ $\{\emptyset, X,\{a\},\{b\}\}$. It can see that $\{a\} \cup\{b\}=\{a, b\} \notin B E S(X, \tau)$.

Theorem 2.8. The intersection of two ${ }^{\star} b$-sets is $a^{\star} b$-set.

Proof: Let $A$ and $B$ two ${ }^{\star} b$-sets, then $A=C l(\operatorname{Int}(A)) \cap \operatorname{Int}(C l(A))$ and $B=C l(\operatorname{Int}(B)) \cap$ $\operatorname{Int}(C l(B))$. Now,

$$
\begin{aligned}
A \cap B & =[C l(\operatorname{Int}(A)) \cap \operatorname{Int}(C l(A))] \cap[C l(\operatorname{Int}(B)) \cap \operatorname{Int}(C l(B))] \\
& =[C l(\operatorname{Int}(A)) \cap C l(\operatorname{Int}(B))] \cap[\operatorname{Int}(C l(A)) \cap \operatorname{Int}(C l(B))] \\
& \subseteq[C l(\operatorname{Int}(A) \cap \operatorname{Int}(B)) \cap \operatorname{Int}(C l(A) \cap C l(B)) \\
& \subseteq[C l(\operatorname{Int}(A \cap B)) \cap \operatorname{Int}(C l(A \cap B)) ;
\end{aligned}
$$

$[C l(\operatorname{Int}(A \cap B)) \cap \operatorname{Int}(C l(A \cap B)) \subseteq[C l(\operatorname{Int}(A)) \cap C l(\operatorname{Int}(B))] \cap[\operatorname{Int}(C l(A)) \cap \operatorname{Int}(C l(B))]=A \cap B$.

Therefore, $A \cap B$ is a ${ }^{\star} b$-set.

Theorem 2.9. If $A$ is $a^{\star} b$-set, then $A$ is $a^{\star} b$-open set.

Proof: Let $A$ be a ${ }^{\star} b$-set, then

$A=C l(\operatorname{Int}(A)) \cap \operatorname{Int}(C l(A))$,

$A \subset C l(\operatorname{Int}(A)) \cap \operatorname{Int}(C l(A))$.

Therefore, $A$ is a ${ }^{\star} b$-open set.

The following example shows that the converse of the above Theorem, it is not always true.

Example 2.4. Let $X=\{a, b, c\}$ and $\tau=\{\emptyset, X,\{a\},\{b\},\{a, b\}\}$. Then, $\{a, b\}$ is $a^{\star} b$-open set, but it is not $a^{\star} b$-set.

Proposition 2.2. Let $(X, \tau)$ be a topological space and $A \subset X$. Then, the following statements hold:

1. If $A$ is $a^{\star} b$-set, then $A$ is a semi-open set.

2. If $A$ is $a^{\star} b$-set, then $A$ is a pre-open set.

3. If $A$ is $a^{\star} b$-set, then $A$ is a b-open set.

Proof:

1. Let $A$ be a ${ }^{\star} b$-set, then $A=C l(\operatorname{Int}(A)) \cap \operatorname{Int}(C l(A)) \subset C l(\operatorname{Int}(A)) \cap(\operatorname{Int}(C l(A)) \subset$ $C l(\operatorname{Int}(A))$. Therefore, $A$ is semi-open.

2. Let $A$ be a ${ }^{\star} b$-set, then $A=C l(\operatorname{Int}(A)) \cap \operatorname{Int}(C l(A)) \subset C l(\operatorname{Int}(A)) \cap(\operatorname{Int}(C l(A)) \subset$ $\operatorname{Int}(C l(A))$. Therefore, $A$ is pre-open.

3. Let $A$ be a ${ }^{\star} b$-set, then $A=C l(\operatorname{Int}(A)) \cap \operatorname{Int}(C l(A)) \subset C l(\operatorname{Int}(A)) \cap(\operatorname{Int}(C l(A)) \subset$ $C l(\operatorname{Int}(A)) \cup \operatorname{Int}(C l(A))$. Therefore, $A$ is $b$-open.

Theorem 2.10. Let $(X, \tau)$ be a topological space and $A \subset X$. If $A$ is $a{ }^{\star} b$-set and $C l(\operatorname{Int}(A)) \subsetneq$ $\operatorname{Int}(C l(A))$, then $A$ is a regular-open set.

Proof: Let $A$ be a ${ }^{\star} b$-set, then $A=C l(\operatorname{Int}(A)) \cap \operatorname{Int}(C l(A))$, since $C l(\operatorname{Int}(A)) \subsetneq \operatorname{Int}(C l(A))$, then $A \in \operatorname{Int}(C l(A))$. Therefore, $A$ is a regular-open set.

The following diagram shows the relations proved in this section:

$b$-open sets $\Longleftarrow$ semi-open sets $\Longleftarrow{ }^{\star} b$-open sets $\Longrightarrow$ pre-open sets $\Longrightarrow b$-open sets.

Now, in this part of the section, it introduces the concept of ${ }^{\star} b$-compact space and it shows some properties.

Definition 2.5. Let $(X, \tau)$ be a topological space and $\mathcal{F}$ be a filter base of $X$. Then $\mathcal{F}$ is said to be ${ }^{\star} b$-convergent to point $x \in X$ if fore every ${ }^{\star} b$-open set $U$ containing $x$, there exits a $F \in \mathcal{F}$ such that $F \subset U$.

Definition 2.6. Let $(X, \tau)$ be a topological space and $\mathcal{F}$ be a filter base of $X$. Then, $\mathcal{F}$ is said to be ${ }^{\star} b$-accumulates to a point $x \in X$ if $F \cap U \neq \emptyset$, for every $\mathcal{F}$-open set $U$ containing in $X$ and every $F \in \mathcal{F}$.

Proposition 2.3. Let $(X, \tau)$ be a topological space, $\mathcal{F}$ be a filter base of $X$ and $A$ is any closed set containing $x$. If there exits a $F \in \mathcal{F}$ such that $F \subset A$. Then, $\mathcal{F}{ }^{\star} b$-converges to a point $x \in X$.

Proof: Let $U$ be any ${ }^{\star} b$-open set containing $x$. Then, for each $x \in U$, there exits a closed set $A$ such that $x \in A \subset U$. By hypothesis, there exits a $F \in \mathcal{F}$ such that $F \subset A \subset U$ which implies that $F \subset U$. Therefore, $\mathcal{F}{ }^{\star} b$-converges to a point $x \in X$.

Proposition 2.4. Let $(X, \tau)$ be a topological space and $\mathcal{F}$ be a filter base and $A$ is any closed set containing $x$ such that $F \cap A \neq \emptyset$ for each $F \in \mathcal{F}$, then $\mathcal{F}$ is ${ }^{\star} b$-accumulation to point $x \in X$.

Proof: The proof is similar to the Proposition 2.3.

Definition 2.7. Let $(X, \tau)$ be a topological space. Then, $X$ is said to be ${ }^{\star} b$-compact if fore every ${ }^{\star}$ b-open cover $\left\{V_{\delta}: \delta \in \Delta\right\}$ of $X$, there exits a finite subset $\Delta_{0}$ of $\Delta$ such that $X=\bigcup\left\{V_{\delta}: \delta \in \Delta_{0}\right\}$

Theorem 2.11. If every closed cover of a topological space $(X, \tau)$ has a finite sub-cover, then $X$ is ${ }^{\star}$ b-compact.

Proof: Let $\left\{V_{\delta}: \delta \in \Delta_{0}\right\}$ be any ${ }^{\star} b$-open cover of $X$ and $x \in X$. Then, for each $x \in V_{\delta}(x), \delta \in \Delta$, there exists a closed set $F_{\delta}(x)$ such that $x \in F_{\delta}(x) \subset V_{\delta}(x)$. Then, the family $\left\{F_{\delta}(x): x \in X\right\}$ is a 
cover of $X$ by closed set. Now, by hypothesis, that family has a finite sub-cover such that $X=\left\{F_{\delta}\left(x_{j}\right)\right.$ : $j=1,2, \ldots, n\} \subset\left\{V_{\delta}\left(x_{j}\right): j=1,2, \ldots, n\right\}$. Hence, $X=\left\{V_{\delta}\left(x_{j}\right): j=1,2, \ldots, n\right\}$. Therefore, $X$ is ${ }^{\star} b$-compact.

3. Generalized of ${ }^{\star} b$-closed sets. In this section, we use the notion of ${ }^{\star} b$-closed sets to introduce the notion of generalized of ${ }^{\star} b$-closed sets.

Definition 3.1. Let $(X, \tau)$ be a topological space and $A \subset X$. Then, $A$ is said to be a generalized ${ }^{\star} b$-closed set or simply $g^{\star} b$-closed set if ${ }^{\star} b-C l(A) \subseteq U$, whenever $A \subseteq U$ and $U$ is a open set. The complement of a $g^{\star} b$-closed set is called generalized ${ }^{\star} b$-open set or simply $g^{\star} b$-open set.

Remark 3.1. The collection of all $g^{\star} b$-closed sets and $g^{\star} b$-open sets are denoted by $G B E(X, \tau)$ and $G B E(X, \tau)$, respectively.

Proposition 3.1. Every ${ }^{\star} b$-closed set is $g^{\star} b$-closed set.

Proof: The proof is followed by the Definition 3.1. The following example shows that the converse of the above Proposition, it is not always true.

Example 3.1. Let $X=\{a, b, c, d\}$ and $\tau=\{\emptyset, X,\{a\},\{b\},\{a, b\},\{a, b, d\}\}$. Then, $\{a, d\}$ is $a$ $g^{\star} b$-closed set, but it is not $a^{\star} b$-closed set.

Theorem 3.1. Let $A$ be a $g^{\star} b$-closed subset of $X$. Then, ${ }^{\star} b-C l(A)-A$ does not contain any non-empty closed sets.

Proof: Let $F$ be a closed set of $X$ such that $F \subseteq \subseteq^{\star} b-C l(A)-A$. Since $X-F$ is a open set, then $A \subseteq X-F$ and $A$ is $g^{\star} b$-closed, it follows ${ }^{\star} b-C l(A) \subseteq X-F$, in consequence $F \subseteq X-{ }^{\star} b-C l(A)$. This implies that $F \subseteq\left(X-{ }^{\star} b-C l(A)\right) \cap\left({ }^{\star} b-C l(A)-A\right)=\emptyset$, therefore $F=\emptyset$. set.

Corollary 3.1. Let $A$ be a $g^{\star} b$-closed set. Then, $A$ is ${ }^{\star} b$-closed if and only if ${ }^{\star} b-C l(A)-A$ is a closed

Proof: Let $A$ be $g^{\star} b$-closed set. If $A$ is ${ }^{\star} b$-closed, it has ${ }^{\star} b-C l(A)-A=\emptyset$ which is a closed set. Conversely, let ${ }^{\star} b-C l(A)-A$ be a closed set. Then, by the Theorem $3.1,{ }^{\star} b-C l(A)-A$ does not contain any non-empty closed set and ${ }^{\star} b-C l(A)$ is a closed set of itself. Thus, ${ }^{\star} b-C l(A)-A=\emptyset$. Therefore, $A={ }^{\star} b-C l(A)$, in consequence $A$ is a ${ }^{\star} b$-closed set.

Corollary 3.2. Let $A$ be an open set and $g^{\star} b$-closed set. Then, $A \cap J$ is $g^{\star} b$-closed set whenever ${ }^{\star} b$ closed set $J$ of $X$. Proof: Since $A$ is $g^{\star} b$-closed and open set, then ${ }^{\star} b-C l(A) \subseteq A$ and so $A$ is a ${ }^{\star} b$-closed. Therefore, $A \cap J$ is ${ }^{\star} b$-closed set of $X$ and this implies that $A \cap J$ is $g^{\star} b$-closed set of $X$.

Theorem 3.2. Let $(X, \tau)$ be a topological space and $A, B \subseteq X$. If $A$ is a $g^{\star} b$-closed set and $B$ is any set such that $A \subseteq B \subseteq{ }^{\star} b-C l(A)$, then $B$ is a $g^{\star} b$-closed set of $X$.

Proof: Let $B \subseteq V$ where $V$ is an open set of $X$. Since $A$ is a $g^{\star} b$-closed set and $A \subseteq V$, then ${ }^{\star} b$ $C l(A) \subseteq V$ and so ${ }^{\star} b-C l(A)={ }^{\star} b-C l(B)$. Therefore, ${ }^{\star} b-C l(B) \subseteq V$ and hence $B$ is a $g^{\star} b$-closed set of $X$.

Theorem 3.3. Let $(X, \tau)$ be a topological space and $A \subset X . A$ is a $g^{\star} b$-open set if and only if $J \subseteq \subseteq^{\star} b$-Int $(A)$ whenever $J$ closed set and $J \subseteq A$.

Proof: Let $A$ be a $g^{\star} b$-open set and let $J \subseteq A$ where $J$ is a closed set. Then, $X-A$ is a $g^{\star} b$-closed set contained in the open set $X-J$. Therefore, ${ }^{\star} b-C l(X-A) \subseteq X-J$ and $X-{ }^{\star} b-\operatorname{Int}(A) \subseteq X-J$. In consequence, $J \subseteq^{\star} b$ - $\operatorname{Int}(A)$.

Conversely, if $A$ is a closed set with $J \subseteq{ }^{\star} b-\operatorname{Int}(A)$ and $J \subseteq A$, then $X-{ }^{\star} b$ - Int $(A) \subseteq X-J$. Therefore, ${ }^{\star} b-C l(X-A) \subseteq X-J$. Hence, $X-A$ is a $g^{\star} b$-closed set and $A$ is a $g^{\star} b$-open set of $X$.

4. Locally ${ }^{\star} b$-closed sets. In this section, we use the notion of ${ }^{\star} b$-closed sets to introduce the notion of locally ${ }^{\star} b$-closed sets.

Definition 4.1.

A subset A of a topological space $(X, \tau)$ is called locally ${ }^{\star} b$-closed if $A=U \cap V$, where $U \in \tau$ an $V$ is $a{ }^{\star} b$-closed.

\section{Remark 4.1.}

The collection of all locally ${ }^{\star} b$-closed sets is denoted by $\operatorname{LCB}(X, \tau)$. The following example shows that the notions of ${ }^{\star} b$-open and locally ${ }^{\star} b$-closed are independent.

Example 4.1. Let $X=\{a, b, c, d\}$ and $\tau=\{\emptyset,\{a\},\{b\},\{a, b\},\{a, b, d\}\}$. Then, $\{a, b\}$ is $a^{\star} b$-open set, but it is not a locally ${ }^{\star} b$-closed set and $\{a, d\}$ is a locally ${ }^{\star} b$-closed set, but it is not $a{ }^{\star} b$-open set.

Theorem 4.1. The intersection of a locally $b$-closed set and a locally ${ }^{\star} b$-closed set is a locally b-closed set.

Proof: Let $C, D \subseteq X$. Then $C$ and $D$ are said to be separated if $C \cap C l(D)=\emptyset$ and $D \cap C l(C)=\emptyset$.

Theorem 4.2. Let $(X, \tau)$ be a topological space and $X$ is extremally disconnected space. For a subset A of $X$, the following statements are equivalent:

1. A is a open set.

2. $A$ is ${ }^{\star} b$-open and locally closed. 
Proof: $(1) \Rightarrow(2)$ : Let $A$ be a open set, then by the Lemma $2.1 A$ is a ${ }^{\star} b$-open set, besides $A \subseteq U$ and $A \subseteq V$, where $U \in \tau$ and $V$ is a closed set, therefore $A=U \cap V$.

$(2) \Rightarrow(1):$ If $A$ is a ${ }^{\star} b$-open set and $A$ is locally closed imply that $A=U \cap V$, where $U \in \tau$, therefore $A \subseteq U$, and so $\operatorname{Int}(A)=\operatorname{Int}(U)$. Moreover, $A \subset^{\star} b-\operatorname{Int}(A)=\operatorname{Int}(A)$. In consequence $A$ is a open set.

Definition 4.2. Let $(X, \tau)$ be a topological space and $A \subset X$. Then, $A$ is said to be $D\left(c,{ }^{\star} b\right)$-set if $\operatorname{Int}(A)={ }^{\star} b$-Int $(A)$. The following example shows that the notions of ${ }^{\star} b$-open and $D\left(c,{ }^{\star} b\right)$-set are independent.

Example 4.2. Let $X=\{a, b, c, d\}$ and $\tau=\{\emptyset,\{a\},\{b\},\{a, b\},\{a, b, d\}\}$. Then, Int $(\{a, b, c\})=$ $\{a, b\}$, but ${ }^{\star} b-\operatorname{Int}(\{a, b, c\})=\{a, b, c\}$.

Theorem 4.3. For a subset $A$ of a space $(X, \tau)$, the following statements are equivalent.

1. A is open.

2. $A$ is ${ }^{\star} b$-open and $D\left(c,{ }^{\star} b\right)$-set.

Proof:

$(1) \Rightarrow(2)$ : If $A$ is a open set, then $A$ is a ${ }^{\star} b$-open set and $A=\operatorname{Int}(A)={ }^{\star} b$ - $\operatorname{Int}(A)$ and so $A$ is $D\left(c,{ }^{\star} b\right)$-set.

$(2) \Rightarrow(1):$ If $A \in B S O(X, \tau)$ and $A \in D\left(c,{ }^{\star} b\right)$ imply that $A={ }^{\star} b-\operatorname{Int}(A)$ and $\operatorname{Int}(A)={ }^{\star} b$ $\operatorname{Int}(A)$, in consequence $A$ is open.

Proposition 4.1. Let $V$ a subset of $(X, \tau), V$ is locally ${ }^{\star} b$-closed if and only if there exits an open set $U \subset X$ such that $V=U \cap^{\star} b-C l(V)$.

Proof: Since $V$ is locally ${ }^{\star} b$-closed, then $V=U \cap J$, where $U$ is an open set an $J$ is a ${ }^{\star} b$-closed set. Thus, $V \subseteq U$ and $V \subseteq J$, then $U \subseteq{ }^{\star} b-C l(V) \subseteq{ }^{\star} b-C l(J)=J$. Therefore, $V \subseteq U \cap^{\star} b-C l(V) \subseteq U \cap^{\star} b$ $C l(J)=U \cap J=V$. In consequence, $V=U \cap^{\star} b-C l(V)$. Conversely, since ${ }^{\star} b-C l(V)$ is ${ }^{\star} b$-closed and $V=U \cap^{\star} b-C l(V)$, then $V$ is locally ${ }^{\star} b$-closed.

Proposition 4.2. Let $(X, \tau)$ be a topological space and $A \subset X$. If $A$ is locally ${ }^{\star} b$-closed, then the following statements hold:

1. ${ }^{\star} b-C l(A)-A$ is ${ }^{\star} b$-closed set.

2. $\left[A \cup\left(X-{ }^{\star} b-C l(A)\right)\right]$ is ${ }^{\star} b$-open.

3. $A \subseteq^{\star} b-\operatorname{Int}\left(A \cup\left(X-{ }^{\star} b-C l(A)\right)\right.$.

Proof:

1. If $A$ i a locally ${ }^{\star} b$-closed, then there exits a $V$ open set such that $A=V \cap^{\star} b-C l(A)$. Now,

${ }^{\star} b-C l(A)-A={ }^{\star} b-C l(A)-\left[V \cap{ }^{\star} b-C l(A)\right]$

$=^{\star} b-C l(A) \cap\left[X-\left(V \cap \cap^{\star} b-C l(A)\right]\right.$

$={ }^{\star} b-C l(A) \cap\left[(X-V) \cup\left(X-{ }^{\star} b-C l(A)\right)\right]$

$=\left[{ }^{\star} b-C l(A) \cap(X-V)\right] \cup\left[{ }^{\star} b-C l(A) \cap\left(X-{ }^{\star} b-C l(A)\right)\right]$

$={ }^{\star} b-C l(A) \cap(X-V)$.

Which is a ${ }^{\star} b$-closed set by the Corollary 2.1 .

2. Since ${ }^{\star} b-C l(A)-A$ is a ${ }^{\star} b$-close set, then $\left[X-\left({ }^{\star} b-C l(A)-A\right)\right]$ is a ${ }^{\star} b$-open set and $\left[X-\left({ }^{\star} b\right.\right.$ $C l(A)-A)]=X-\left(\left({ }^{\star} b-C l(A) \cap(X-A)\right)=\left[A \cup\left(X-{ }^{\star} b-C l(A)\right)\right]\right.$.

3. It is obvious that $A \subseteq\left[A \cup\left(X-{ }^{\star} b-C l(A)\right)\right]={ }^{\star} b-\operatorname{Int}\left[A \cup\left[A \cup\left(X-{ }^{\star} b-C l(A)\right)\right]\right.$.

Corollary 4.1. The intersection of a locally ${ }^{\star} b$-closed set and locally set is locally ${ }^{\star} b$-closed.

Proof: The proof is similar to the Theorem 4.1.

Theorem 4.4. Suppose that $(X, \tau)$ is under finite union of ${ }^{\star} b$-closed sets. Let $A$ and $B$ be locally ${ }^{\star} b$-closed. If $A$ and $B$ are separated, then $A \cup B$ is locally ${ }^{\star} b$-closed.

Proof: Since $A$ and $B$ are locally ${ }^{\star} b$-closed, then $A=Q \cap^{\star} b-C l(A)$ and $B=W \cap^{\star} b-C l(B)$, where $Q, W$ are open set of $X$. Now, put $U=Q \cap(X-C l(B))$ and $V=W \cap(X-C l(A))$. So, $U \cap^{\star} b$ $C l(A)=(Q \cap(X-C l(B))) \cap^{\star} b-C l(A)=A \cap(X-C l(B))=A$, since $A \subseteq X-C l(B)$. Similarly, $V \cap^{\star} b-C l(B)=B$ and $U \cap^{\star} b-C l(B) \subseteq U \cap C l(B)=\emptyset$ and $V \cap^{\star} b-C l(A) \subseteq V \cap C l(A)=\emptyset$. Since $U$ and $V$ are open, it has

$(U \cap V) \cap^{\star} b-C l(A \cup B)=(U \cup V) \cap\left({ }^{\star} b-C l(A) \cup{ }^{\star} b-C l(B)\right)$

$=\left(U \cap^{\star} b-C l(A)\right) \cup\left(U \cap^{\star} b-C l(B)\right) \cup\left(V \cap^{\star} b-C l(A)\right) \cup\left(V \cap^{\star} b-C l(B)\right)$

$=A \cup B$.

Therefore, $A \cup B$ is locally ${ }^{\star} b$-closed.

Theorem 4.5. Let $(X, \tau)$ be a topological space and $A \subseteq X$. Then, the following statements are equivalent:

1. A is ${ }^{\star} b$-closed.

2. $A$ is $g^{\star} b$-closed and locally ${ }^{\star} b$-closed.

Proof: Let $A$ be ${ }^{\star} b$-closed and so $A=A \cap X$, then $A$ is locally ${ }^{\star} b$-closed. If $A \subseteq V$, then ${ }^{\star} b$ $C l(A)=A \subseteq V$ and $A$ is $g^{\star} b$-closed. Conversely, If $A$ is locally ${ }^{\star} b$-closed, then there exits an open set $V$ such that $A=V \cap^{\star} b-C l(A)$, since $A \subseteq V$ and $A$ is $g^{\star} b$-closed, then ${ }^{\star} b-C l(A) \subseteq V$. Therefore, ${ }^{\star} b-C l(A) \subseteq V \cap^{\star} b-C l(A)=A$. In consequence, $A$ is ${ }^{\star} b$-closed. 
Definition 4.3. Let $(X, \tau)$ be a topological space and $A \subset X$. Then $A$ is said to be:

1. ${ }^{\star} b$-t-set if $\operatorname{Int}(A)=\operatorname{Int}\left({ }^{\star} b-C l(A)\right)$.

2. ${ }^{\star} b$ - $B$-set if $A=U \cap V$, where $U \in \tau$ and $V$ is $a^{\star} b$ - $t$-set

Proposition 4.3. Let $(X, \tau)$ be a topological space and $A, B \subseteq X$. Then the following statements hold:

1. If $A$ is ${ }^{\star} b$-closed, then $A$ is ${ }^{\star} b$ - $t$-set.

2. If $A$ and $B$ are ${ }^{\star} b$-t-sets, then $A \cap B$ is $a^{\star} b$-t-set.

Proof:

1. Let $A$ be a ${ }^{\star} b$-closed set, then $A={ }^{\star} b-C l(A)$ and $\operatorname{Int}(A)=\operatorname{Int}\left({ }^{\star} b-C l(A)\right)$, therefore $A$ is a ${ }^{\star} b$ - $t$-set.

2. Let $A$ and $B$ be ${ }^{\star} b$ - $t$-sets. Then, it has

$\operatorname{Int}(A \cap B) \subseteq \operatorname{Int}\left({ }^{\star} b-C l(A \cap B)\right)$

$\subseteq\left(\operatorname{Int}\left({ }^{\star} b-C l(A)\right) \cap\left({ }^{\star} b-C l(B)\right)\right)$

$=\operatorname{Int}\left({ }^{\star} b-C l(A) \cap \operatorname{Int}\left({ }^{\star} b-C l(B)\right)\right.$

$=\operatorname{Int}(A) \cap \operatorname{Int}(B)$

$=\operatorname{Int}(A \cap B)$.

Therefore, $\operatorname{Int}(A \cap B)=\operatorname{Int}\left({ }^{\star} b-C l(A \cap B)\right)$, hence $A \cap B$ is a ${ }^{\star} b$ - $t$-set.

The converse of the part (1) of the above proposition, it is not always true as the following example shows.

Example 4.3. Let $X=\{a, b, c, d\}$ and $\tau=\{\emptyset, X,\{a\},\{b\},\{a, b\},\{a, b, d\}\}$. Then, $\{a\}$ is $a{ }^{\star} b$ - $t$-set, but it is not $a^{\star} b$-closed set. The following example shows that the union of two ${ }^{\star} b$ - $t$-sets not need be a ${ }^{\star} b$ - $t$-set.

Example 4.4. Let $X=\{a, b, c, d\}$ and $\tau=\{\emptyset, X,\{a\},\{b\},\{a, b\},\{a, b, d\}\}$. Then the collection of all ${ }^{\star} b$-t-sets $=\{\emptyset, X,\{b, c, d\},\{a, c, d\},\{c, d\},\{c\},\{d\},\{a\},\{b\},\{b, c\},\{a, c\},\{a, d\},\{b, d\}\}$. Now, it can see that $\{a\} \cup\{b\}=\{a, b\}$ is not $a^{\star} b$-t-set.

Lemma 4.1. Let $(X, \tau)$ be a topological space and $A \subset X$. If $A$ is $a^{\star} b$-t-set, then $A$ is $a{ }^{\star} b$ - $B$-set. Proof: The proof is followed by the Definition 4.3 .

The following diagram shows the relations proved in the sections three and four:

$g^{\star} b$-closed and locally ${ }^{\star} b$-closed $\Longleftrightarrow{ }^{\star} b$-closed sets $\Longrightarrow g^{\star} b$-closed sets.

5. Regular generalized ${ }^{\star} b$-closed set. In this section, we use the notion of ${ }^{\star} b$-closed sets to introduce the notion of regular generalized of ${ }^{\star} b$-closed sets.

Definition 5.1. Let $(X, \tau)$ be a topological space and $A \subseteq X$. Then, $A$ is said to be regular generalized ${ }^{\star} b$-closed or simply $\mathrm{rg}^{\star} b$-closed set if ${ }^{\star} b-C l(A) \subseteq U$ whenever $A \subseteq U$ and $U$ is a regular open set of $X$. The complement of a $r g^{\star} b$-closed set is called $r g^{\star} b$-open set.

Remark 5.1. The collection of all $r g^{\star} b$-closed sets and $r g^{\star} b$-open sets are denoted by $R G E C(X, \tau)$ and $R G E O(X, \tau)$, respectively.

Lemma 5.1. Let $A$ be a subset of a topological space $(X, \tau)$. Then, ${ }^{\star} b-C l(A) \subseteq C l(A)$. Proof: The proof is clear.

Theorem 5.1. Every closed set is $r g^{\star} b$-closed set.

Proof: Let $B$ be any closed set of $X$ such that $B \subseteq V$. where $V$ is a regular open set. Since ${ }^{\star} b$ $C l(B) \subseteq C l(B)=B$. Therefore, ${ }^{\star} b-C l(B) \subseteq V$. In consequence, $B$ is a $r g^{\star} b$-closed set. The following example shows that the converse of the above Theorem need not be true.

Example 5.1. Let $X=\{a, b, c, d\}$ and $\tau=\{\emptyset, X,\{a\},\{b\},\{a, b\},\{a, b, c\},\{b, c\}\}$. Then, $\{b, c\}$ is $a$ $r g^{\star} b$-closed set, but it is not a closed set.

Theorem 5.2. Every ${ }^{\star} b$-closed set is $r g^{\star} b$-closed set.

Proof: Let $B$ be any ${ }^{\star} b$-closed set of $X$ such that $V$ is any regular open set containing $B$. Since $B$ is a ${ }^{\star} b$-closed set, then ${ }^{\star} b-C l(B)=B$. Therefore, ${ }^{\star} b-C l(B) \subseteq V$. Hence, $B$ is a $r g^{\star} b$-closed set. The following example shows that the converse of the above theorem need not be true.

Example 5.2. Let $X=\{a, b, c, d\}$ and $\tau=\{\emptyset, X,\{a\},\{b\},\{a, b\},\{a, b, c\},\{b, c\}\}$. Then, $\{a, c\}$ is $a$ $r g^{\star} b$-closed set, but it is not $a^{\star} b$-closed set.

Theorem 5.3. Every $r g^{\star} b$-closed set is gpr-closed set.

Proof: Let $B$ be any closed set of $X$ and $V$ be any regular open set containing $B$. Since every ${ }^{\star} b$-open set is pre-open set, then ${ }^{\star} b-C l(A) \subseteq p C l(A) \subseteq V$. Therefore, ${ }^{\star} b-C l(B) \subseteq V$. Hence, $B$ is a $g p r$-closed set. The following example shows that the converse of the above theorem need not be true.

Example 5.3. Let $X=\{a, b, c\}$ and $\tau=\{\emptyset, X,\{a\},\{b\},\{a, b\}\}$. Then, $\{a, b\}$ is a gpr-closed set, but it is not a $\mathrm{rg}^{\star} b$-closed set.

Theorem 5.4. Every $r g^{\star} b$-closed set is $r g b$-closed set.

Proof: Let $B$ be any closed set of $X$ and $V$ be any regular open set containing $B$. Since every ${ }^{\star} b$-open set is $b$-open set, then ${ }^{\star} b-C l(A) \subseteq b C l(A) \subseteq V$. Therefore, ${ }^{\star} b-C l(B) \subseteq V$. Hence, $B$ is a $r g b$-closed set. 
The following example shows that the converse of the above Theorem need not be true.

Example 5.4. Let $X=\{a, b, c\}$ and $\tau=\{\emptyset, X,\{a\},\{b\},\{a, b\}\}$. Then, $\{b, c\}$ is a rgb-closed set, but it is not a $r g^{\star} b$-closed set.

Theorem 5.5. Every $r g^{\star} b$-closed set is $g^{\star} b$-closed set.

Proof: Let $B$ a $r g^{\star} b$-closed set of $X$ such that $V$ is any regular open set containing $B$. Since every regular open set is a open set, then ${ }^{\star} b-C l(B) \subseteq V$. Therefore, $A$ is a $g^{\star} b$-closed set of $X$. The following example shows that the converse of the above Theorem need not be true.

Example 5.5. Let $X=\{a, b, c\}$ and $\tau=\{\emptyset, X,\{a\},\{b\},\{a, b\}\}$. Then, $\{a, b\}$ is a $g^{\star} b$-closed set, but it is not a rg*b-closed set.

Definition 5.2. Let $(X, \tau)$ be a topological space and $A \subseteq X$. Then, $A$ is said to be generalized $\alpha^{\star} b$-closed set or simply $g \alpha^{\star} b$-closed set if ${ }^{\star} b-C l(A) \subseteq U$ whenever $A \subseteq U$ and $U$ is a $\alpha$-open set of $X$. The complement of a $g \alpha^{\star} b$-closed set is a $g \alpha^{\star} b$-open set.

Theorem 5.6. Every $r g^{\star} b$-closed set is $g \alpha^{\star} b$-closed set.

Proof: Let $B$ be a $r g^{\star} b$-closed set of $X$ such that $V$ is any regular open set containing $B$. Since every regular open is $\alpha$-open, then ${ }^{\star} b-C l(A) \subseteq V$ and $A \subseteq V$, where $U$ be $\alpha$-open. Therefore, $A$ is a $g \alpha^{\star} b$-closed set. The following example shows that the converse of the above Theorem need not be true.

Example 5.6. Let $X=\{a, b, c\}$ and $\tau=\{\emptyset, X,\{a\}\}$. Then, $\{a, b\}$ is a ga $\alpha^{\star} b$-closed set, but it is not a $r g^{\star} b$-closed set.

Definition 5.3. Let $(X, \tau)$ be a topological space and $A \subseteq X$. Then, $A$ is said to be semi generalized ${ }^{\star} b$-closed set or simply sg ${ }^{\star} b$-closed set if ${ }^{\star} b-C l(A) \subseteq U$ whenever $A \subseteq U$ and $U$ is a semi-open set of $X$.

Theorem 5.7. Every $r g^{\star} b$-closed set is $s g^{\star} b$-closed set.

Proof: Let $B$ be a $r g^{\star} b$-closed set of $X$ such that $V$ is any regular open set containing $B$. Since every regular open is semi-open, then ${ }^{\star} b-C l(A) \subseteq V$ and $A \subseteq V$, where $U$ be semi-open. Therefore, $A$ is a $s g^{\star} b$-closed set. The following example shows that the converse of the above Theorem need not be true.

Example 5.7. Let $X=\{a, b, c\}$ and $\tau=\{\emptyset, X,\{a\},\{b\},\{a, b\}\}$. Then, $\{a, c\}$ is a sg $g^{\star} b$-closed set, but it is not a $\mathrm{rg}^{\star} b$-closed set.

6. Weakly ${ }^{\star} b$-closed set. In this section, we use the notion of ${ }^{\star} b$-closed sets to introduce the notion of weakly of ${ }^{\star} b$-closed sets.

Definition 6.1. Let $(X, \tau)$ be a topological space and $A \subset X$. Then, $A$ is said to be weakly ${ }^{\star} b$-closed if $C l(\operatorname{Int}(A)) \subseteq U$ whenever $A \subseteq U$ and $U$ is $a^{\star} b$-closed set of $X$. The complement of a weakly ${ }^{\star} b$-closed is called weakly ${ }^{\star}$ b-open set.

Proposition 6.1. For a topological spaces $(X, \tau)$ the following statements hold:

1. Every ${ }^{\star} b$-closed set is weakly ${ }^{\star} b$-closed set.

2. Every closed set is weakly ${ }^{\star} b$-closed set.

3. Every nowhere dense set $B$ of $X$ is weakly ${ }^{\star} b$-closed.

Proof:

1. Let $B$ be a ${ }^{\star} b$-closed set and let $V$ be a ${ }^{\star} b$-open set containing $B$. Now, since $B$ is ${ }^{\star} b$-closed set, it has that $A=\operatorname{In}(C l(A)) \cap C l(\operatorname{Int}(A)) \subseteq C l(\operatorname{Int}(A)) \subseteq V$. Therefore, $B$ is weakly ${ }^{\star} b$-closed set.

2. Let $B$ be any closed set and let $V$ be a ${ }^{\star} b$-open set containing $B$. Now, since $B$ is a closed set, it has that $B=C l(B) \subseteq C l(\operatorname{Int}(B)) \subseteq V$. Therefore, $B$ is weakly ${ }^{\star} b$-closed set.

3. Since $\operatorname{Int}(B) \subseteq \operatorname{Int}(C l(B))$ and $B$ is nowhere dense. Now, by the Definition 1.9, $\operatorname{Int}(B)=\emptyset$, therefore $C l(\operatorname{Int}(B))=\emptyset$. Hence, $B$ is a weakly ${ }^{\star} b$-closed set.

The following example shows that the converse of part (1) of the above Proposition, it is not always true.

Example 6.1. Let $V=[2,4]$ and $B=\{3\}$, Then, $B \subseteq V$ and $C l(\operatorname{Int}(B))=\emptyset \subseteq V$. Therefore, $B$ is weakly ${ }^{\star} b$-closed set, but it is not $a{ }^{\star} b$-closed set. The following example shows that the converse of part (2) of the above Proposition, it is not always true.

Example 6.2. Let $X=\{a, b, c, d\}$ and $\tau=\{\emptyset, X,\{a\},\{b\},\{a, b\}\}$. Then, $\{c\}$ is a weakly ${ }^{\star} b$-closed set, but it is not a closed set.

Proposition 6.2. Let $(X, \tau)$ be a topological space and $A \subset X$. If $B$ is both open and weakly ${ }^{\star} b$-closed set, then $B$ is a closed set.

Proof: Sine $B$ is both open and weakly ${ }^{\star} b$-closed, then $C l(B)=C l(\operatorname{Int}(B)) \subseteq B$. Therefore, $B$ is a closed set.

Theorem 6.1. Let $(X, \tau)$ be a topological space and $A \subset X . B$ is weakly ${ }^{\star} b$-closed if and only if $C l(\operatorname{Int}(B))-B$ contains non-empty ${ }^{\star} b$-closed set in $X$.

Proof: Let $B$ be a weakly ${ }^{\star} b$-closed set of $X$ and let $V$ be a ${ }^{\star} b$-closed set such that $V \subseteq C l(\operatorname{int}(B))-$ $B$. Now, since $X-V$ is a ${ }^{\star} b$-open set and $B \subseteq X-V$, then $C l(\operatorname{Int}(B)) \subseteq X-V$ and that is $V \subseteq$ 
$X-(C l(\operatorname{Int}(B)))$, this implies that $V \subseteq C l(\operatorname{Int}(B)) \cap(X-(C l(\operatorname{Int}(B))))=\emptyset$. Conversely, let $B \subseteq V$ where $V$ is a ${ }^{\star} b$-open set of $X$. Now, if $C l(\operatorname{Int}(B))$ is not contained in $V$, then $C l(\operatorname{Int}(B)) \cap(X-V)$ is a non-empty ${ }^{\star} b$-closed set of $C l(\operatorname{Int}(B))-B$ and this is a contradiction.

Theorem 6.2. If $A$ is a weakly ${ }^{\star} b$-closed set and $A \subseteq B \subseteq C l(\operatorname{Int}(A))$. Then, $B$ is weakly ${ }^{\star} b$-closed set.

Proof: Since $A \subseteq B$, it has that $C l(\operatorname{int}(B))-B \subseteq C l(\operatorname{int}(A))-A$. Now, by the Theorem 6.1, $C l(\operatorname{Int}(A))-A$ contains no non-empty ${ }^{\star} b$-closed set. Thus, $C l(\operatorname{Int}(B))-B$ contains no non-empty ${ }^{\star} b$-closed set and so by the Theorem $6.1, B$ is a weakly ${ }^{\star} b$-closed set.

Theorem 6.3. Let $(X, \tau)$ be a topological space and $A \subset X$. Then, $B$ is weakly ${ }^{\star} b$-open set if and only if $W \subseteq \operatorname{Int}(C l(B))$ whenever $W \subseteq B$ and $W$ is weakly ${ }^{\star} b$-closed set.

Proof: Let $B$ be any weakly ${ }^{\star} b$-open set of $X$. Then, $X-B$ is a weakly ${ }^{\star} b$-closed set. Now, let, $V$ be a ${ }^{\star} b$-closed set contained $B$. Then, $X-B$ is a ${ }^{\star} b$-open set of $X$ containing $X-B$. Since $X-B$ is a weakly ${ }^{\star} b$-closed, it has $C l(\operatorname{int}(B)) \subseteq X-V$. Therefore, $V \subseteq \operatorname{Int}(C l(B))$. Conversely, suppose that $V \subseteq \operatorname{Int}(C l(B))$ whenever $V \subseteq B$ and $V$ is a ${ }^{\star} b$-closed set of $X$. Then, $X-V$ is a ${ }^{\star} b$-open set containing $X-B$ and $X-V \supseteq C l(\operatorname{Int}(\bar{X}-B))$. Therefore, $X-B$ is a weakly ${ }^{\star} b$-closed set, hence $B$ is a weakly ${ }^{\star} b$-open set.

7. Conclusion. In this article some generalizations of *b-closed sets were presented, from the results obtained, we could observe some existing inclusion relationships between these notions, additionally, those inclusions that are not satisfied, examples are presented.

With the results obtained in this article, new inclusion relationships can be extended and presented with some existing sets in the literature.

ORCID and License

Carlos Granados https://orcid.org/0000-0002-7754-1468

This work is licensed under the Creative Commons - Attribution 4.0 International (CC BY 4.0)

\section{References}

[1] Al-Omari A, Salmi M. Decomposition of continuity via b-open set. Bol. Soc. Paran. Mat. 1996; 26(1-2): 53-64.

[2] Andrijivic D. On $b$-open sets. Mat. Vesnik. 1996; 48: 59-64.

[3] Gnanambal Y. On generalized pre-regular closed sets in topological spaces. Indian J. Pure Appl. Math. 1997; 28: 351-360.

[4] Granados C. A decomposition of continuity and contra continuity in ideal nano topological spaces. Journal of the international Mathematical Virtual Institute. 2020; 10(2): 271-286.

[5] Granados C. A decomposition of open sets through operator interior, International Journal of Advances in Applied Mathematics and Mechanics. 2020; 8(2): 15-26.

[6] Granados C. A new notion of convergence on ideal topological spaces, Selecciones Matemáticas. 2020; 7(2): 250-256.

[7] Hariwan Z. Bc-open sets in topological spaces. Advances in pure Mathematics. 2013; 3: 34-40.

[8] Hdeib H. $\omega$-closed mappings. Revista Colombiana de Mat. 1982; 16: 65-78.

[9] Indira T, Rekha K. On locally ${ }^{\star \star} b$-closed sets. Proceedings of the Heber International Conference on Applications of Mathematics an Statistics. 2012.

[10] Kuratowski K. Topologie I. Monografie Matematyczne tom 3. PWN-Polish Scientific Publishers. Warszawa. 1933.

[11] Levine N. Semi-open sets and semi-continuity in topological spaces. Armer Math. Monthly. 1963; 70: 36-41.

[12] Mariappa K, Sekar S. On regular generalized b-closed set. Int. Journal of Math. Analysis. 2013; 3(13): 613-624.

[13] Mashhour A, El-monsef M, El-deeb S. On pre-continuous and weak pre-continuous mappings. Proc. Math. Phys. Soc. Egypt. 1982; 53:47-53.

[14] Noiri T, Keskin A. On $\Lambda_{I}$-sets and some weak separation axioms. Int. J. Math. Anal. 2011; 5(11): 539-548.

[15] Stone M. Applications of the theory of Boolean rings to general topology. Trans. Armer. Math. Soc. 1937; 41:375-381.

[16] Usha R, Thangavelu P. On locally numeralb-closed sets and weakly numeralb-closed sets. South Asian Journal Of Multidisciplinary Studies. 2015; 2(3):181-196. 